\title{
Regional Gradient Exact Enlarge Controllability of the Semilinear Heat Equation
}

\author{
Touria Karite $^{\text {}}$, Ali Boutoulout ${ }^{2}$, and Fatima Zahrae EL Alaoui ${ }^{3}$ \\ 123 TSI Team, MACS Laboratory, Institute of Sciences \\ Moulay Ismail University, Meknes, Morocco \\ ${ }^{1}$ touria.karite@gmail.com, ${ }^{2}$ boutouloutali@yahoo.fr, ${ }^{3}$ fzelalaoui2011@yahoo.fr
}

\begin{abstract}
The aim of this paper is to study the enlarge gradient-controllability problem of the heat equation. The purpose is to compute the control $u$ which steers the state gradient of the system between two prescribed levels $l_{1}$ and $l_{2}$, only on a subregion $\omega$ of the system evolution domain $\Omega$. The obtained results have been proved via two approaches, the sub-differential and Lagrangian multiplier approach.
\end{abstract}

Keywords: Distributed systems, parabolic systems, regional controllability, gradient, sub-differential, Lagrangian approach, semilinear systems, minimum energy, heat equation.

\section{Introduction}

The regional controllability concept refers to control problems in which the target of interest is not fully specified as a state, but refers only to a region $\omega$ of the spatial domain $\Omega$ on which the governing partial differential system (1) is considered. In many real-world dynamic systems, this regional idea occurs naturally when it comes to modeling natural phenomenon just in a specific region. For example, when we want to keep the temperature of a place or a device between two bounds $T_{1}$ and $T_{2}$. This concept has been widely developed and interesting results have been proved, in particular, the possibility to reach a state only on an internal subregion $\omega$ of $\Omega$ [ or on a part of the boundary $\partial \Omega$ of $\Omega[2$.

Later the concept of internal regional gradient controllability was developed [3]. It consists of steering the state gradient of system (1) to a prescribed function only given on $\omega$ located in the interior of $\Omega$. Here instead of steering the system to a desired gradient, we are interested in steering its gradient between two prescribed functions given only on a subregion $\omega$ in the interior of $\Omega$. This problem is both practical and beneficial to study and this is due to the fact that we may be only interested in the knowledge of the states in a critical subregion of the whole domain. And also it is more realistic and more adapted for system analysis than the classical one since, in general, the dynamic of a physical process isn't really well represented by a mathematical model of a real system, and there can be many reasons for this: the parameters may not be known precisely, the model can be of reduced order, also, in order to simplify the calculations, the model can be a linear approximation of a nonlinear process, and since the calculated control is based only on an approximate model, the final state of the original system will not be exact. Besides in numerical calculations and because of rounding errors, a computed solution can be far from the actual one. Therefore, the solutions of such systems are known approximately as well as their gradients.

There are many reasons motivating the study of this problem which is the enlarge controllability or also called controllability with constraints on the state 4]: first, the mathematical model of a real system is obtained from measurements or from approximation techniques and it is very often affected by perturbations. Consequently, the

${ }^{*}$ Corresponding author.Touria KARITE ${ }^{1}$ touria.karite@gmail.com 2010 Mathematics Subject Classification: 93B05, 93C10, 93C20. 
solution of such system is approximately known, and second, in various real problems the target is required to be between two bounds. This is the case, for example, of a nuclear reactor in which the temperature of the water circulating around the reactor should not exceed a certain level. Many works were subject to study the problem in the parabolic case [5] or the hyperbolic for linear systems [6].

This paper is an extension of the previous ones and it deals with the controllability properties of semilinear heat equation where the control $u$ is exerted in an intern subregion $\omega$ of the system evolution domain $\Omega$. Thus, let $\Omega$ be an open bounded set of $\mathbb{R}^{n}(n \geq 1)$ with regular boundary $\partial \Omega$. We consider the Banach spaces $\mathcal{H}=L^{2}(\Omega)$ and $\mathcal{V}=H_{0}^{1}(\Omega)$, and the corresponding norms $\|\cdot\|_{\mathcal{H}}$ and $\|\cdot\|_{\mathcal{V}}$. For a given $T>0$, we denote $\left.Q_{T}=\Omega \times\right] 0, T[$, $\left.\Sigma_{T}=\partial \Omega \times\right] 0, T\left[\right.$ and let us consider the following problem : Minimize $\mathrm{J}(\mathrm{u})=\frac{\alpha}{2}\left\|\chi_{\omega} \nabla y_{u}(T)-y_{d}\right\|_{\mathcal{V}}^{2}+\frac{\beta}{2}\|u\|_{U}^{2}$ subject to: $\begin{cases}\partial_{t} y(x, t)-\Delta y(x, t)-\mathcal{N} y(x, t)=B u(t) & Q_{T} \\ y(x, 0)=y_{0}(x) & \Omega, \\ y(\xi, t)=0 & \Sigma_{T},\end{cases}$

- $\alpha$ and $\beta$ are two positive constants in $\mathbb{N}$,

- $\chi_{\omega}$ the restriction operator defined by:

$$
\begin{aligned}
\chi_{\omega}:(\mathcal{H})^{n} & \longrightarrow\left(L^{2}(\omega)\right)^{n} \\
y_{i} & \longmapsto \chi_{\omega} y_{i}=\left.y_{i}\right|_{\omega},
\end{aligned}
$$

while $\omega \in \Omega$ is of Lebesgue positive measure.

- $\nabla$ is nabla operator given by the formula:

$$
\begin{aligned}
\nabla: \mathcal{V} & \longrightarrow(\mathcal{H})^{n} \\
y & \longmapsto \nabla y=\left(\frac{\partial y}{\partial x_{1}}, \ldots, \frac{\partial y}{\partial x_{n}}\right),
\end{aligned}
$$

- $y_{u}(T)$ is the mild solution of (1) at the time $T\left(y_{u}(T) \in \mathcal{H}[7]\right), y_{d}$ is the desired state in $\mathcal{V}$ and $u$ is a the control function in the control space $U=L^{2}\left(0, T ; \mathbb{R}^{m}\right)$ (where $m$ is the number of actuators),

- $\Delta$ is the Laplacian operator with dense domain and it is an infinitesimal generator of a $C_{0}$-semigroup $(S(t))_{t \geq 0}$ on $\mathcal{H}$ which we consider compact,

- $\mathcal{N}: L^{2}(0, T ; \mathcal{H}) \rightarrow \mathcal{H}$ is a non linear $k$-Lipschitz operator [8, 9],

- $B \in \mathcal{L}\left(\mathbb{R}^{m}, \mathcal{H}\right)$ and $y_{0}$ is the initial datum in $\mathcal{H}$.

The problem (1) is well-posed and has a unique solution.

The remainder contents of this paper are structured as follows. Some definitions, properties and some notations are introduced in the next section and in section 3, the proof of the existence and the uniqueness of the solution of problem (1) is given. Two approaches to compute the control $u$ are used in section 4 .

\section{Gradient enlarge controllability}

Without loss of generality, we denote by $y_{u}($.$) the mild solution of (1) when it is excited by a control u$, it is obtained using the idea of Duhamel's principle. The final solution at time $T$ could be written as follows:

$$
y_{u}(T)=S(T) y_{0}+\int_{0}^{T} S(T-t) \mathcal{N} y(t) d t+\int_{0}^{T} S(T-t) B u(t) d t .
$$

We first introduce the closed sub-vectorial space $F$ of $\mathcal{H}$. The problem of gradient exact enlarge controllability (depending on $F$ ) is defined as follows [10]:

Definition 2.1. Given $T>0$. We say that there is gradient exact enlarge controllability, if, for every $y_{0}$ (in a suitable functional space), we can find a control $u$ such that

$$
\chi_{\omega} \nabla y(., T ; u) \in F
$$


Remark 2.2. $\quad$ 1. This notion depends of course on the choice of the functional space $F$.

2. If $F=\{0\}$, we retrieve the classical notion of the exact controllability.

For the particular case, we will study the gradient enlarge controllability just in $[a(),. b()$.$] . For that let us consider$ $(a(.))_{i}$ and $(b(.))_{i}$ be two given real functions in $\left(L^{2}(\omega)\right)^{n}$ such that $(a(.))_{i} \leq(b(.))_{i}$ for $i=1, \ldots, n$ a.e in $\omega$, and we set:

$$
\gamma=[a(.), b(.)]=\left\{\left(y_{1}, \ldots, y_{n}\right) \in\left(L^{2}(\omega)\right)^{n} \quad \mid \quad a_{i}(.) \leq y_{i}(.) \leq b_{i}(.) \quad \text { a.e on } \omega \text { for all } i \in\{1, \ldots, n\}\right\} .
$$

so the definition of the gradient enlarge controllability in $\gamma$ is the following:

Definition 2.3. We say that (1) is $\gamma$-gradient controllable in $\omega$ at time $T$ if there exists a command $u \in U$ such that:

$$
\chi_{\omega} \nabla y_{u}(T) \in \gamma
$$

Let us define some operators:

- The Duhamel operator $H$ from $U$ to $\mathcal{V}$ such that for $u \in U$ :

$$
H u=\int_{0}^{T} S(T-s) B u(s) d s,
$$

- and the operator $G_{\gamma}$ given by:

$$
\begin{aligned}
G_{\gamma}: L^{2}(0, T ; \mathcal{V}) & \longrightarrow \mathcal{V} \\
y(.) & \longmapsto \int_{0}^{T} S(T-\tau) \mathcal{N} y(\tau) d \tau
\end{aligned}
$$

Finally, the solution of the system (1) at the time $T$ could be written as follow:

$$
y_{u}(T)=S(T) y_{0}+G_{\gamma} y+H u
$$

We have the following proposition:

Proposition 2.4. We say that system (1) is $\gamma$-gradient controllable in $\omega$ at time $T$ if and only if:

$$
\left(\operatorname{Im} \chi_{\omega} \nabla G_{\gamma}+\operatorname{Im} \chi_{\omega} \nabla H\right) \cap \gamma \neq \emptyset .
$$

Proof. We suppose that the system (1) is $\gamma$-gradient controllable in $\omega$ at time $T$ which is equivalent to write: $\chi_{\omega} \nabla y_{u}(T) \in \gamma$.

We know that:

$$
\begin{aligned}
\chi_{\omega} \nabla y_{u}(T) & =\chi_{\omega} \nabla\left(S(T) y_{0}+G_{\gamma} y+H u\right) \\
& =\chi_{\omega} \nabla S(T) y_{0}+\chi_{\omega} \nabla G_{\gamma} y+\chi_{\omega} \nabla H u .
\end{aligned}
$$

We have $\nabla S(T) y_{0}=0$, we denote by: $z_{1}=\chi_{\omega} \nabla G_{\gamma} y, z_{2}=\chi_{\omega} \nabla H u$ and $z=z_{1}=\chi_{\omega} \nabla y_{u}(T)$. This leads to: $z_{1} \in \operatorname{Im}\left(\chi_{\omega} \nabla G_{\gamma}\right)$ and $z_{2} \in \operatorname{Im}\left(\chi_{\omega} \nabla H\right)$. Thus, $z \in \operatorname{Im}\left(\chi_{\omega} \nabla G_{\gamma}\right)+\operatorname{Im}\left(\chi_{\omega} \nabla H\right)$ and $z \in \gamma$ which gives:

$$
\left(\operatorname{Im} \chi_{\omega} \nabla G_{\gamma}+\operatorname{Im} \chi_{\omega} \nabla H\right) \cap \gamma \neq \emptyset .
$$

Conversely, we suppose that the following expression is true:

$$
\left(\operatorname{Im} \chi_{\omega} \nabla G_{\gamma}+\operatorname{Im} \chi_{\omega} \nabla H\right) \cap \gamma \neq \emptyset,
$$

then, there exists $z \in \gamma$ such that $z \in\left(\operatorname{Im} \chi_{\omega} \nabla G_{\gamma}+\operatorname{Im} \chi_{\omega} \nabla H\right)$. So $z=z_{1}+z_{2}$ where $z_{1}=\chi_{\omega} \nabla G_{\gamma} y$ with $y \in L^{2}\left(0, T ;(H)^{n}\right)$ and $\exists u \in \mathcal{U}$ such that $z_{2}=\chi_{\omega} \nabla H u$. Then $z=\chi_{\omega} \nabla G_{\gamma} y+\chi_{\omega} \nabla H u$.

While $\chi_{\omega} \nabla S(T) y_{0}=0$ we could be write:

$$
\begin{aligned}
z & =\chi_{\omega} \nabla S(T) y_{0}+\chi_{\omega} \nabla G_{\gamma} y+\chi_{\omega} \nabla H u \\
& =\chi_{\omega} \nabla\left(S(T) y_{0}+G_{\gamma} y+H u\right) \\
& =\chi_{\omega} \nabla y_{u}(T) .
\end{aligned}
$$

Finally, $z=\chi_{\omega} \nabla y_{u}(T) \in \gamma$, and we prove the equivalence. 
Remark 2.5. $\quad$ 1. The above definition means that we are interested in the transfer of the system (1) to an unknown state just in $\gamma$.

2. If $\gamma=\{0,0\}$ or $a()=.b($.$) we retrieve the regional exact controllability. So, for a(.) \neq b($.$) the \gamma$-gradient controllability constitutes an extension of regional controllability.

We can also characterize the enlarge controllability by using the notion of strategic actuators. Hence, we recall that an actuator is conventionally defined by a couple $(D, f)$, where $D$ is a nonempty closed part of $\bar{\Omega}$ representing the geometric support of the actuator, and $f \in L^{2}(D)$ define the spatial distribution of the action on the support $D$.

Definition 2.6. The actuator $(D, f)$ is said to be $\gamma$-strategic on $\omega$ if the excited system is $\gamma$-gradient controllable in $\omega$.

In the case of a pointwise actuator (internal or boundary) $D=\{b\}$ and $f=\delta(b-$.), where $\delta$ is the Dirac mass concentrated in $b$, and the actuator is then denoted by $\left(b, \delta_{b}\right)$. For definitions and properties of strategic actuators we refer to [11, 12.

\section{$3 \quad$ Existence and uniqueness}

In this section we prove the existence and uniqueness of the solution of the problem (1), for that we consider the following problem:

$$
\inf _{u \in \mathcal{U}_{a d}} J(u)
$$

where $\mathcal{U}_{a d}=\left\{u \in U \mid \chi_{\omega} \nabla y_{u}(T)=y_{d} \in \gamma\right\}$ is the set of admissible directions. Hence the problem (1) becomes

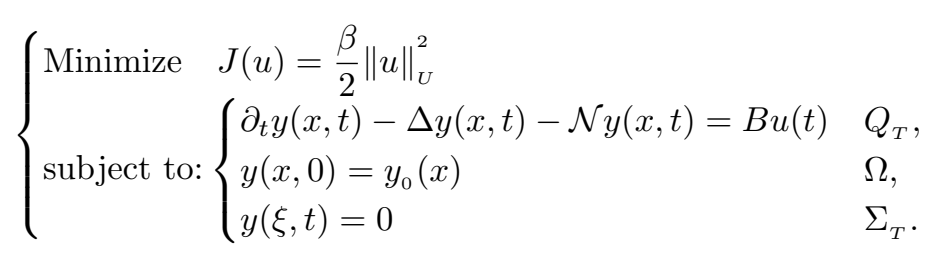

The following result prove the existence of the solution for problem (3).

Proposition 3.1. There exists $u^{*} \in \mathcal{U}_{a d}$ solution of problem (3).

Proof. The set $\mathcal{K}=\left\{J(u) \mid u \in \mathcal{U}_{a d}\right\}$ is nonempty and nonnegative then it has a nonnegative infimum.

Let $\left(u_{n}\right)_{n \in \mathbb{N}}$ be a minimizing sequence in $\mathcal{U}_{a d}$. From the problem (1) we can see that:

$$
\left\|u_{n}\right\|_{U}^{2} \leq \frac{2}{\beta} J\left(u_{n}\right)
$$

then $\left(u_{n}\right)_{n \in \mathbb{N}}$ is bounded.

Thus, there exists a subsequence of $\left(u_{n}\right)_{n \in \mathbb{N}}$ denoted also $\left(u_{n}\right)_{n \in \mathbb{N}}$ which converges weakly to $u^{*}$.

The set $\mathcal{U}_{a d}$ is closed and convex, so $u^{*} \in \mathcal{U}_{a d}$. And we have,

$$
\begin{aligned}
y_{u_{n}}(t)-y_{u^{*}}(t) & =\int_{0}^{t} S(t-s) \mathcal{N} y_{u_{n}}(s) d s+\int_{0}^{t} S(t-s) B u_{n}(s) d s \\
& -\int_{0}^{t} S(t-s) \mathcal{N} y_{u^{*}}(s) d s-\int_{0}^{t} S(t-s) B u^{*}(s) d s \\
& =\int_{0}^{t} S(t-s) \mathcal{N}\left(y_{u_{n}}(s)-y_{u^{*}}(s)\right) d s \\
& +\int_{0}^{t} S(t-s) B\left(u_{n}(s)-u^{*}(s)\right) d s .
\end{aligned}
$$

So

$$
\left|y_{u_{n}}(t)-y_{u^{*}}(t)\right| \leq \int_{0}^{t}\|S(t-s)\| k\left|y_{u_{n}}(s)-y_{u^{*}}(s)\right| d s+\left|\int_{0}^{t} S(t-s) B\left(u_{n}(s)-u^{*}(s)\right) d s\right| \cdot
$$


$\mathcal{N}$ is a $k$-Lipschitz non linear operator $\left(\left|\mathcal{N}\left(y_{u_{n}}(t)-y_{u^{*}}(t)\right)\right| \leq k\left|y_{u_{n}}(t)-y_{u^{*}}(t)\right|\right)$, and using the Gronwall lemma, we have:

$$
\left|y_{u_{n}}(t)-y_{u^{*}}(t)\right| \leq e^{\int_{0}^{t} k\|S(t-s)\| d s}\left|\int_{0}^{t} S(t-s) B\left(u_{n}(s)-u^{*}(s)\right) d s\right| .
$$

Using the property of semi-group $[8]$, there exists $M \geq 1, \rho \in \mathbb{R}$ such that $\|S(t)\| \leq M e^{\rho t}$, then we obtain:

$$
\left|y_{u_{n}}(t)-y_{u^{*}}(t)\right| \leq e^{k M e^{\rho t} t^{1 / 2}}\left|\int_{0}^{t} S(t-s) B\left(u_{n}(s)-u^{*}(s)\right) d s\right| .
$$

By theorem (3.9) in [13, the weak convergence $u_{n} \rightarrow u^{*}$ implies $B u_{n} \rightarrow B u^{*}$ weakly in $L^{2}(0, T ; \mathcal{H})$ with $n>1$.

We know that the semi-group $(S(t))_{t \geq 0}$ is compact, using the corollary (3.3) in chapter 3 in [14] we have:

$$
\lim _{n \rightarrow \infty} \sup _{t \in[0, T]}\left|\int_{0}^{t} S(t-s) B\left(u_{n}(s)-u^{*}(s)\right) d s\right|=0 .
$$

It follows from (4) that $y_{u_{n}} \rightarrow y_{u^{*}}$ strongly in $L^{2}(0, T ; \mathcal{H})$.

Using the uniform continuity of $\left(y_{u_{n}}\right)_{n}$ and also the continuity of the restriction operator we obtain:

$$
\lim _{n \rightarrow \infty}\left\|\chi_{\Gamma} \gamma_{0} y_{u_{n}}(T)-y_{d}\right\|^{2}=\left\|\chi_{\Gamma} \gamma_{0} y_{u^{*}}(T)-y_{d}\right\|^{2} .
$$

Then $J\left(u^{*}\right) \leq \lim _{n \rightarrow \infty} \inf J\left(u_{n}\right)$, because $\left\|u^{*}\right\| \leq \lim _{n \rightarrow \infty} \inf \left\|u_{n}\right\|$ and $\left(u_{n}\right)_{n}$ is weakly convergent. Thus, we obtain: $J\left(u^{*}\right)=\inf _{u \in \mathcal{U}_{a d}} J\left(u_{n}\right)$.

For the uniqueness property we have the following proposition:

Proposition 3.2. The system (1) is $\gamma$-gradient controllable in $\omega$, then the problem (3) have a unique solution $u^{*} \in \mathcal{U}_{a d}$.

Proof. If the system (1) is $\gamma$-gradient controllable in $\omega$, we have $\chi_{\omega} \nabla y_{u}(T) \in \gamma$, which implies that $\mathcal{U}_{\text {ad }} \neq \emptyset$. If $u \in \mathcal{U}_{a d}$ then $J(u)=\frac{\beta}{2}\|u\|^{2}$ and since the mapping $f: u \mapsto \frac{1}{2}\|u\|^{2}$ is strictly convex, coercive $\left(\lim _{\|u\| \rightarrow+\infty} f(u)=+\infty\right.$ for $u \in U$ ), proper and lower semi-continuous in $U$ (which is reflexive), we verify that $\mathcal{U}_{a d}$ is closed convex subset of $U$.

It is obvious that $\mathcal{U}_{a d}$ is convex, indeed, for $(u, v) \in \mathcal{U}_{a d}^{2}$ and $t \in[0,1]$ :

$$
\chi_{\omega} \nabla y_{(1-t) u+t v}(T) \in \gamma .
$$

To prove that $\mathcal{U}_{a d}$ is closed, we consider a sequence $\left(u_{n}\right)_{n}$ in $\mathcal{U}_{a d}$ such that $u_{n} \rightarrow u$ strongly in $U$. Since $\chi_{\omega} \nabla H$ and $\chi_{\omega} \nabla G_{\gamma}$ are both continuous then: $\chi_{\omega} \nabla H u_{n} \rightarrow \chi_{\omega} \nabla H u$ strongly in $\left(L^{2}(\omega)\right)^{n}$.

Hence $\chi_{\omega} \nabla y_{u}(T) \in \gamma$ where $\gamma$ is a closed subset.

Then $\chi_{\omega} \nabla y_{u}(T)=\chi_{\omega} \nabla\left(S(T) y_{0}+G_{\gamma} y+H u_{n}\right) \in \gamma$. So $\chi_{\omega} \nabla y_{u}(T) \in \gamma$, which means that $u \in \mathcal{U}_{a d}$. Thus we prove that $\mathcal{U}_{\text {ad }}$ is closed. Then (2) admits a unique solution $u^{*}$.

\section{Computation of the control}

In order to compute the control subject to our problem, we will use the sub-differential analysis. For that, we consider the problem (1) with the constant $\beta=1$.

\subsection{Sub-differential approach}

Let $f$ be a nontrivial, lower semi-continuous, proper and convex function from a Hilbert space $W$ to $\widetilde{R}=]-\infty,+\infty[$. We denote by $\mathcal{F}(W)$ the set of the functions $f$, $\|$.$\| is the Hilbert norm of W$.

- For $f \in \mathcal{F}(W)$, let $\operatorname{dom}(f)=\{u \in W \mid f(u)<\infty\}$ and $f^{*}$ is the polar function of $f$. 
- For $f \in \mathcal{F}(W)$ and $\lambda$ a positive parameter, there exists a unique solution $J_{\lambda}$ [15] of the problem: $f_{\lambda}(x):=$ $\inf _{y \in W}\left\{f(y)+\frac{1}{2 \lambda}\|y-x\|^{2}\right\}$, such that:

$$
f_{\lambda}(x)=\left\{f\left(J_{\lambda} x\right)+\frac{1}{2 \lambda}\left\|J_{\lambda} x-x\right\|^{2}\right\} .
$$

This solution could be characterized by the following variational inequality:

$$
\forall y \in W, \quad \frac{1}{\lambda}\left\langle J_{\lambda} x-x, J_{\lambda} x-y\right\rangle+f\left(J_{\lambda} x\right)-f(y) \leq 0 .
$$

- For $v_{0} \in \operatorname{dom}(f)$, the set

$$
\partial f\left(v_{0}\right)=\left\{u^{*} \in W \mid f(u) \geq f\left(v_{0}\right)+\left\langle u^{*}, u-v_{0}\right\rangle \quad \forall u \in W\right\},
$$

denotes the sub-differential of $f$ at $v_{0}$.

With all these notations, the problem (2) is equivalent to:

$$
\left\{\begin{array}{l}
\inf \left(\sigma+\Psi_{u_{a d}}\right)(u) \\
u \in \mathcal{U}_{a d},
\end{array}\right.
$$

where:

- $\Psi_{K}(u)= \begin{cases}0 & \text { if } u \in K \\ +\infty & \text { otherwise }\end{cases}$ denotes the indicator functional of $K$ ( $K$ a non empty subset of $W$ ).

- $\sigma(u)=\frac{1}{2}\|u\|^{2}$.

Hence the solution of (5) is characterized by the following result.

Proposition 4.1. $u^{*}$ is a solution of (2) if and only of if the system (1) is $\gamma$-gradient controllable in $\omega$ and:

$$
u^{*} \in \mathcal{U}_{a d} \quad \text { and } \quad \Psi_{\mathcal{U}_{a d}}^{*}\left(-u^{*}\right)=-\left\|u^{*}\right\|^{2} .
$$

Proof. System (1) is $\gamma$-gradient controllable in $\omega$, then $\mathcal{U}_{a d} \neq \emptyset$. Using Fermat's rule, we have $u^{*}$ is a minimum of 22 if and only if $0 \in \partial\left(\sigma+\Psi_{\mathcal{u}_{a d}}\right)\left(u^{*}\right)$. Although, $\mathcal{U}_{a d}$ is convex and $\sigma \in \mathcal{F}(U)$ so since $\mathcal{U}_{a d}$ is closed, convex and non empty, we have $\Psi_{u_{a d}} \in \mathcal{F}(U)$

Moreover, $\operatorname{dom} \sigma \cap \operatorname{dom} \Psi_{u_{a d}} \neq \emptyset$ because the system $(1)$ is $\gamma$-gradient controllable in $\omega$.

Furthermore, $\partial\left(\sigma+\Psi_{u_{a d}}\right)\left(u^{*}\right)=\partial \sigma\left(u^{*}\right)+\partial \Psi_{u_{a d}}\left(u^{*}\right)\left(\sigma\right.$ is continuous). It follows that $u^{*}$ is a solution of 22 if and only if

$$
0 \in \partial \sigma\left(u^{*}\right)+\partial \Psi_{u_{a d}}\left(u^{*}\right)
$$

But we have $\sigma$ is Frechet-differentiable, so $\partial \sigma\left(u^{*}\right)=\left\{u^{*}\right\}$. Then $u^{*}$ is the solution of (2) if and only if $-u^{*} \in$ $\partial \Psi_{u_{a d}}\left(u^{*}\right)$ which is equivalent to $\Psi_{u_{a d}}\left(u^{*}\right)+\Psi_{u_{a d}}^{*}\left(-u^{*}\right)=-\left\|u^{*}\right\|^{2}$. We conclude that $u^{*} \in \mathcal{U}_{a d}$ and $\Psi_{\mathfrak{u}_{a d}}^{*}\left(-u^{*}\right)=$ $-\left\|u^{*}\right\|^{2}$.

We consider: $\widetilde{\gamma}=[\widetilde{a}(),. \widetilde{b}()$.$] where:$

$$
\begin{aligned}
& \widetilde{a}(.)=a(.)-\chi_{\omega} \nabla S(T) y_{0} \\
& \text { and } \quad \widetilde{b}(.)=b(.)-\chi_{\omega} \nabla S(T) y_{0}
\end{aligned}
$$

Then, $\mathcal{U}_{a d}=\left\{u \in \mathcal{U} \mid \chi_{\omega} \nabla H u \in \widetilde{\gamma}\right\}$. We have the following result:

Proposition 4.2. $u^{*}$ is the solution of (2) if and only if

$$
\inf _{y \in \widetilde{\gamma}}\left\{u^{*},\left(\chi_{\omega} \nabla H\right)^{\dagger} y\right\}=\left\|u^{*}\right\|^{2}
$$

where $\left(\chi_{\omega} \nabla H\right)^{\dagger}=\left(\chi_{\omega} \nabla H\right)^{*}\left(\left(\chi_{\omega} \nabla H\right)\left(\chi_{\omega} \nabla H\right)^{*}\right)^{-1}$ is the generalized inverse of $\left(\chi_{\omega} \nabla H\right)$.

Moreover, the system (1) is $\gamma$-gradient controllable in $\omega$. 
Proof. We have $\mathcal{U}_{a d}=\left(\chi_{\omega} \nabla H\right)^{\dagger} \widetilde{\gamma}$, from the previous proposition 4.1), $u^{*}$ is the solution of the problem (2) if and only if $u^{*} \in \mathcal{U}_{a d}$ and $\Psi_{\mathcal{U}_{a d}}^{*}\left(-u^{*}\right)=-\left\|u^{*}\right\|^{2}$. Then, we have

$$
\begin{aligned}
\Psi_{\mathcal{u}_{a d}}^{*}\left(v^{*}\right) & =\sup _{v \in \mathcal{U}_{a d}}\left\langle v^{*}, v\right\rangle \quad \forall v^{*} \in \mathcal{U} \\
& =\sup _{v \in\left(\chi_{\omega} \nabla H\right)^{\dagger} \widetilde{\gamma}}\left\langle v^{*}, v\right\rangle \\
& =\sup _{y \in \widetilde{\gamma}}\left\langle v^{*},\left(\chi_{\omega} \nabla H\right)^{\dagger} y\right\rangle \\
& =\sup _{\lambda \in[0,1]}\left\langle\left(\left(\chi_{\omega} \nabla H\right)^{\dagger}\right)^{*} v^{*}, \lambda \widetilde{a}(.)+(1-\lambda) \widetilde{b}(.)\right\rangle
\end{aligned}
$$

Since the mapping $\lambda \mapsto\left\langle\left(\left(\chi_{\omega} \nabla H\right)^{\dagger}\right)^{*} v^{*}, \lambda \widetilde{a}()+.(1-\lambda) \widetilde{b}().\right\rangle$ is convex and continuous from $[0,1]$ to $\mathbb{R}$, we obtain according to Krein-Milman theorem [16]:

$$
\Psi_{u_{a d}}^{*}\left(v^{*}\right)=\sup _{\lambda \in[0,1]}\left\langle\left(\left(\chi_{\omega} \nabla H\right)^{\dagger}\right)^{*} v^{*}, \lambda \widetilde{a}(.)+(1-\lambda) \widetilde{b}(.)\right\rangle .
$$

It follows that:

$$
\Psi_{u_{a d}}^{*}\left(v^{*}\right)=\max \left\{\left\langle v^{*},\left(\chi_{\omega} \nabla H\right)^{\dagger} \widetilde{a}(.)\right\rangle,\left\langle v^{*},\left(\chi_{\omega} \nabla H\right)^{\dagger} \widetilde{b}(.)\right\rangle\right\} .
$$

So we obtain (7) from (6).

Remark 4.3. If $a()=.b()=.\left\{y_{d}\right\}$, then

$$
\widetilde{a}(.)=\widetilde{b}(.)=y_{d}-\chi_{\omega} \nabla S(T) y_{0}-\chi_{\omega} \nabla G_{\gamma} y,
$$

and

This implies that

$$
\mathcal{U}_{a d}=\left(\chi_{\omega} \nabla H\right)^{\dagger}\left(y_{d}-\chi_{\omega} \nabla S(T) y_{0}-\chi_{\omega} \nabla G_{\gamma} y\right)
$$

$$
u^{*}(t)=\left(\chi_{\omega} \nabla H\right)^{\dagger}\left(y_{d}-\chi_{\omega} \nabla S(T) y_{0}-\chi_{\omega} \nabla G_{\gamma} y\right),
$$

if it exists.

\subsection{Lagrangian approach}

We consider the problem (2) when the system (1) is excited by one actuator. The following result gives a useful characterization of the solution of the problem.

Theorem 4.4. If the system (1) is $\gamma$-gradient controllable in $\omega$ then the solution of (2) is given by:

$$
u^{*}=-\left(\chi_{\omega} \nabla H\right)^{*} \lambda^{*}
$$

where $\lambda^{*} \in L^{2}(\omega)$ satisfies:

$$
\left\{\begin{array}{l}
z^{*}=P_{\gamma}\left(\rho \lambda^{*}+z^{*}\right) \\
R_{\gamma} \lambda^{*}+z^{*}=\chi_{\omega} \nabla\left[S(T) y_{0}+G_{\gamma} y\right],
\end{array}\right.
$$

while $P_{\gamma}:\left(L^{2}(\omega)\right)^{n} \rightarrow \gamma$ denotes the projection operator, $\rho>0$ and $R_{\gamma}=\left(\chi_{\omega} \nabla H\right)\left(\chi_{\omega} \nabla H\right)^{*}$.

Proof. If the system (1) is $\gamma$-gradient controllable in $\omega$ then $\mathcal{U}_{a d} \neq \emptyset$ and the problem (2) has a unique solution. Problem (2) is equivalent to the saddle point problem:

$$
\left\{\begin{array}{l}
\inf \frac{1}{2}\|u\|^{2} \\
(u, z) \in Z,
\end{array}\right.
$$


where $Z=\left\{(u, z) \in \mathcal{U} \times \gamma \mid \chi_{\omega} \nabla y_{u}(T)-z=0\right\}$.

To study this constraint, we will use a Lagrangian functional and steer the problem (10) to a saddle point problem. We associate to the problem (10) the Lagrangian functional defined by

$$
\forall(u, z, \lambda) \in \mathcal{U} \times \gamma \times\left(L^{2}(\omega)\right)^{n}, L(u, z, \lambda)=\frac{1}{2}\|u\|^{2}+\left\langle\lambda, \chi_{\omega} \nabla y_{u}(T)-z\right\rangle .
$$

$\triangleright$ We prove that $L$ admits a saddle point.

The set $\mathcal{U} \times \gamma$ is non empty, closed and convex. The functional $L$ satisfies conditions:

- $(u, z) \mapsto L(u, z, \lambda)$ is convex and lower semi-continuous for all $\lambda \in\left(L^{2}(\omega)\right)^{n}$.

- $\lambda \mapsto L(u, z, \lambda)$ is concave and upper semi-continuous for all $(u, z) \in \mathcal{U} \times \gamma$.

Moreover, there exists $\lambda_{0} \in\left(L^{2}(\omega)\right)^{n}$ such that

$$
\lim _{\|(u, z)\| \rightarrow+\infty} L\left(u, z, \lambda_{0}\right)=+\infty
$$

and there exists $\left(u_{0}, z_{0}\right) \in \mathcal{U} \times \gamma$ such that

$$
\lim _{\|\lambda\| \rightarrow+\infty} L\left(u_{0}, z_{0}, \lambda\right)=-\infty .
$$

Using the theorem 1 in [17 the functional $L$ admits a saddle point.

$\triangleright$ We prove then that $u^{*}$ is a solution of $(2)$ and it is the minimum one:

Let $\left(u^{*}, z^{*}, \lambda^{*}\right)$ be a saddle point of $L$. Hence, we have:

$$
L\left(u^{*}, z^{*}, \lambda\right) \leq L\left(u^{*}, z^{*}, \lambda^{*}\right) \leq L\left(u, z, \lambda^{*}\right) \quad \forall(u, z, \lambda) \in \mathcal{U} \times \gamma \times\left(L^{2}(\omega)\right)^{n} .
$$

From the first inequality of (14) we have:

$$
\left\langle\lambda, \chi_{\omega} \nabla y_{u^{*}}(T)-z^{*}\right\rangle \leq\left\langle\lambda^{*}, \chi_{\omega} \nabla y_{u^{*}}(T)-z^{*}\right\rangle \quad \forall \lambda \in\left(L^{2}(\omega)\right)^{n},
$$

which implies $\chi_{\omega} \nabla y_{u^{*}}(T)=z^{*}$ and hence $\chi_{\gamma} \nabla y_{u^{*}}(T) \in \gamma$.

The second inequality of (14) means that for all $u \in \mathcal{U}$ and $z \in \gamma$, we have:

$$
\frac{1}{2}\left\|u^{*}\right\|^{2}+\left\langle\lambda^{*}, \chi_{\omega} \nabla y_{u^{*}}(T)-z^{*}\right\rangle \leq \frac{1}{2}\|u\|^{2}+\left\langle\lambda^{*}, \chi_{\omega} \nabla y_{u}(T)-z\right\rangle \quad \forall(u, z) \in \mathcal{U} \times \gamma .
$$

Since $\chi_{\omega} \nabla y_{u^{*}}(T)=z^{*}$, it follows that:

$$
\frac{1}{2}\left\|u^{*}\right\|^{2} \leq \frac{1}{2}\|u\|^{2}+\left\langle\lambda^{*}, \chi_{\omega} \nabla y_{u}(T)-z\right\rangle \quad \forall(u, z) \in \mathcal{U} \times \gamma .
$$

Taking $z=\chi_{\omega} \nabla y_{u}(T) \in \gamma$, we obtain:

$$
\frac{1}{2}\left\|u^{*}\right\|^{2} \leq \frac{1}{2}\|u\|^{2}
$$

which implies that $u^{*}$ is of minimum energy.

The following assumptions hold, if $\left(u^{*}, z^{*}, \lambda^{*}\right)$ is a saddle point of $L$ :

$$
\begin{gathered}
\left\langle u^{*}, u-u^{*}\right\rangle+\left\langle\lambda^{*}, \chi_{\omega} \nabla H\left(u-u^{*}\right)\right\rangle=0 \quad \forall u \in \mathcal{U}, \\
-\left\langle\lambda^{*}, z-z^{*}\right\rangle \geq 0 \quad \forall z \in \gamma, \\
\left\langle\lambda-\lambda^{*}, \chi_{\omega} \nabla y_{u^{*}}(T)-z^{*}\right\rangle=0 \quad \forall \lambda \in\left(L^{2}(\omega)\right)^{n} .
\end{gathered}
$$

For more details about the saddle point and its theory, we refer to [18, 19, 20].

From 15 we deduce 8 .

The equation (17) is equivalent to:

$$
\chi_{\omega} \nabla y_{u^{*}}(T)=z^{*}
$$


Since $y_{u^{*}}(T)=S(T) y_{0}+G_{\gamma} y()+.H u^{*}$, we have:

$$
\chi_{\omega} \nabla\left[S(T) y_{0}+G_{\gamma} y(.)+H u^{*}\right]-z^{*}=0 .
$$

Then $\chi_{\omega} \nabla\left[S(T) y_{0}+G_{\gamma} y().\right]+\chi_{\omega} \nabla H u^{*}=z^{*}$.

With (8), we have:

$$
\chi_{\omega} \nabla\left[S(T) y_{0}+G_{\gamma} y(.)\right]-\left(\chi_{\omega} \nabla H\right)\left(\chi_{\omega} \nabla H\right)^{*} \lambda^{*}=z^{*},
$$

with $R_{\gamma}=\left(\chi_{\omega} \nabla H\right)\left(\chi_{\omega} \nabla H\right)^{*}$, we obtain the first equation of $(9)$.

From inequality (16), we obtain:

$$
\left\langle\left(\rho \lambda^{*}+z^{*}\right)-z^{*}, z-z^{*}\right\rangle_{H^{1 / 2}(\Gamma)} \leq 0 \quad \forall z \in \gamma, \rho>0,
$$

which is equivalent to the second equation of $(9)$.

Corollary 4.5. If the system (1) is exactly gradient controllable in $\omega$ and $\rho$ suitably chosen, then the system (9) has only one solution $\left(\lambda^{*}, z^{*}\right)$.

Proof.

The gradient exact controllability in $\omega$ implies that $\left(\chi_{\omega} \nabla H\right)^{*}$ and $R_{\gamma}$ are bijective. So if $\left(u^{*}, z^{*}, \lambda^{*}\right)$ is a saddle point of $L$ then the system (9) is equivalent to

$$
\left\{\begin{array}{l}
R_{\gamma} \lambda^{*}+z^{*}=\chi_{\omega} \nabla\left[S(T) y_{0}+G_{\gamma} y(.)\right] \\
z^{*}=P_{\gamma}\left[\left(-\rho R_{\gamma}^{-1} z^{*}+\rho R_{\gamma}^{-1} \chi_{\omega} \nabla\left(S(T) y_{0}+G_{\gamma} y(.)\right)\right)+z^{*}\right] .
\end{array}\right.
$$

It follows that $z^{*}$ is a fixed point of the function

$$
\begin{aligned}
F_{\rho}: \quad \gamma \quad \longrightarrow \gamma^{\gamma} & \left.\longrightarrow\left(-\rho R_{\gamma}^{-1} y+\rho R_{\gamma}^{-1} \chi_{\omega} \nabla\left(S(T) y_{0}+G_{\gamma} y(.)\right)\right)+y\right] .
\end{aligned}
$$

The operator $R_{\Gamma}$ is coercive, i.e.

$$
\exists m>0 \quad \text { such that }\left\langle R_{\gamma}^{-1} y, y\right\rangle \geq m\|y\|^{2} \quad \forall y \in\left(L^{2}(\omega)\right)^{n} .
$$

It follows that

$$
\begin{aligned}
\left\|F_{\rho}(y)-F_{\rho}(z)\right\| & \leq\left\|-\rho R_{\gamma}^{-1}(y-z)+(y-z)\right\|^{2} \\
& \leq \rho^{2}\left\|R_{1^{-1}}^{2}\right\|^{2}\|y-z\|^{2}+\|y-z\|^{2}-2 \rho\left\langle R_{\gamma}^{-1}(y-z),(y-z)\right\rangle \\
& \leq \rho^{2}\left\|R_{\gamma}^{-1}\right\|^{2}\|y-z\|^{2}+\|y-z\|^{2}-2 \rho m\|y-z\|^{2} \\
& \leq\left(1+\rho^{2}\left\|R_{\gamma}^{-1}\right\|^{2}-2 \rho m\right)\|y-z\|^{2} \quad \forall y, z \in \gamma
\end{aligned}
$$

and we deduce that if $0<\rho<\frac{2 m}{\left\|R_{\gamma}^{-1}\right\|^{2}}$, then $F_{\rho}$ is a contraction mapping. This implies the uniqueness of $z^{*}$ and $\lambda^{*}$.

\section{Remark 4.6.}

If $a()=.b($.$) , we obtain the notion of exact gradient controllability in \omega$, and the solution of (2) is given by:

$$
u^{*}=\left(\chi_{\omega} \nabla H\right)^{*} R_{\gamma}^{-1}\left[b(.)-\chi_{\omega} \nabla\left(S(T) y_{0}+R_{\gamma} y(.)\right)\right] \cdot
$$

\section{Conclusion}

We developed an extension of the regional controllability to a situation encountered in many real problems where we are interested in the knowledge of the states in a critical subregion of the whole domain. Various open questions are still under consideration. The problem of constrained control as well as the case of fractional-order distributed parameter dynamic systems are of great interest.

\section{ACKNOWLEDGEMENTS.}

The work has been carried out with a grant from Hassan II Academy of Sciences and Technology. 


\section{References}

[1] A. El Jai, A. J. Pritchard, M. C. Simon, and E. Zerrik, "Regional controllability of distributed systems", Int. J. Control, Vol.62, No.6, (1995), pp.1351-1365.

[2] E. Zerrik, A. El Jai, and A. Boutoulout, "Actuators and regional boundary controllability of parabolic system", Int. J. Systems Sci., Vol.31, No.1, (2000), pp.73-82.

[3] E. Zerrik, A. Boutoulout and A. Kamal, "Regional gradient controllability of parabolic systems", Int. J. Appl. Math. Comput. Sci., Vol.9, (1999), pp.767-787.

[4] J. L. Lions, "Sur la contrôlabilité exacte élargie",Partial Differential Equations and the Calculus of Variations Vol. 1 of the series Progress in Nonlinear Differential Equations and Their Applications, (1989), pp.703-727.

[5] E. Zerrik and F. Ghafrani, "Minimum energy control subject to output constraints numerical approach", IEE Proc.-Control Theory Appl., Vol.149, No.1, (2002), pp.105-110.

[6] A. Boutoulout, H. Bourray, F. Z. El Alaoui, L. and Ezzahri, "Constrained Controllability for Distributed Hyperbolic Systems", Math. Sci. Lett., Vol.3, No.3, (2014), pp.207-214.

[7] J. L. Lions and E. Magenes, Problèmes aux limites non homogènes et applications, Dunod, 1, (1968).

[8] A. Pazy, Semigroups of Linear Operators and Applications to Partial Differential Equations, Springer-Verlag, New York, (1990).

[9] E. Zeidler, Applied functional analysis : Applications to mathematical physics, Springer-Verlag, New York, (1995).

[10] J. L. Lions, Sur la contrôlabilité exacte élargie, Partial Differential Equations and the Calculus of Variations Vol. 1 of the series Progress in Nonlinear Differential Equations and Their Applications, (1989) pp.703-727.

[11] A. El Jai and A. J. Pritchard, Sensors and actuators in distributed systems analysis, Wiley, New York, (1988).

[12] E. Zerrik, A. El Jai and A. Boutoulout, "Actuators and regional boundary controllability of parabolic system", Int. J. Syst. Sci., Vol.31, No.1, (2000), pp.73-82.

[13] H. Brézis, Analyse fonctionnelle : Théorie et Applications, Masson, Paris, (1983).

[14] X. Li and J. Yong, Optimal Control Theory for Infinite Dimensional Systems, Systems \& Control: Foundations \& Applications, Birkhuser Basel, (1995).

[15] J. P. Aubin, L'analyse non linéaire et ses motivations économiques, Paris, Masson, (1984).

[16] K. Yosida, Functional analysis, Springer-Verlag, Berlin Heidelberg New York, (1980).

[17] A. Matei, "Weak solvability via Lagrange multipliers for two frictional contact models", Annals of the university of Bucharest (mathematical series) Vol.4, (LXII), (2013), pp.179-191.

[18] F. Brezzi and M. Fortin, Mixed and Hybrid Finite Element Methods, Springer-Verlag, New York, (1991).

[19] M. Fortin and R. Glowinski, Augmented Lagrangian Methods: Applications to the numerical solution of boundary-value problems, North-Holland, Vol.15, (1983).

[20] R. T. Rockafellar, "Lagrange multipliers and optimality", SIAM Review, Vol.35, No.2, (1993), pp.183-238. 